\title{
Does help structures play a role in reducing the variation of dwell time in IPSA planning for gynaecological brachytherapy application?
}

\author{
Swamidas Jamema, MSc', Umesh Mahantshetty', DD Deshpande', Smriti Sharma², SK Shrivastava \\ Department of Medical Physics, Tata Memorial Hospital, Mumbai, India, 2Atomic Energy Regulatory Board, India
}

\begin{abstract}
Purpose: To report our experience of dosimetric comparison of IPSA and manual plans, with a focus on the use of help structures (HS) during optimization.

Material and methods: 33 patients who underwent MR image-based HDR intracavitary-brachytherapy for cervix cancer based on GYN-ESTRO recommendations were selected for evaluation. Tandem/ovoid (T/O) and Vienna applicators were used. HS of diameter of $5 \mathrm{~mm}$ were drawn around the tandem/needles/ovoid and ring. Three plans were generated: manual optimized plan (MOPT), IPSA without help structures (IPSA_woHS) and IPSA with help structures (IPSA_wHS). Dose-volume parameters and the loading pattern were evaluated.

Results: For T/O, the use of HS did not make significant impact in the dose-volume parameters and in the loading of tandem and ovoids, however steep variation was found in the individual dwell time. In case of Vienna applicator, inclusion of HS in the optimization made a significant impact in loading of needles. The percentage ratio of total time of needles to the tandem $\left(\mathrm{T}_{\mathrm{N} / \mathrm{T} \%}\right)$ was found to be $14 \pm 2.5,53 \pm 9,22 \pm 6$ for MOPT, IPSA_woHS and IPSA_wHS, respectively, which implies that in IPSA_woHS the dwell time in needles were half of the dwell time in the tandem, while in MOPT the needles were loaded only in $14 \%$, and in IPSA_wHS it was $22 \%$ of the dwell time of tandem. Inclusion of HS in the optimization has reduced the contribution of dwell time of needle in IPSA_wHS. The individual variation of dwell time was also reduced in IPSA_wHS, however drawing of HS is a time consuming procedure and may not be practical for a routine practice.

Conclusion: The role of HS was evaluated for IPSA for T/O and Vienna-applicator, the use of HS may be beneficial in case of combined intracavitary - interstitial approach.

Key words: image based brachytherapy, inverse planning, IPSA.

\begin{abstract}
Purpose
The use of inverse planning algorithms in external beam therapy is an established procedure and widely accepted in the clinics, while in brachytherapy, it is still in the investigational stage, not well accepted, and practiced as yet. Traditionally, in intracavitary brachytherapy, standard loading patterns were used without any optimization, which produced excellent clinical results [1]. Later on, with the introduction of remote afterloaders, standard loading or manual optimization methods were employed which continued to produce similar or better results in terms of local control and toxicities [2,3]. Manual optimization allows the user to change the dwell weights manually or drag the isodose lines using a mouse such that the target coverage is adequate with maximal sparing of OARs. It is a trial and error method where the user has to keep changing the dwell weights
\end{abstract}

until an optimal solution is met. Although it is a time consuming manual process, the dose distribution had the typical quality that did not deviate very much from the standard loading pattern. The traditional pear shaped dose distribution was maintained, except for a few minor changes that improve the target coverage and OAR sparing. On the other hand, the inverse optimization is based on mathematical algorithm where clinical objectives are defined as mathematical equations, the optimal solution is then obtained by minimizing the objective function through an iterative process. The use of inverse planning is popularized with its application for prostate brachytherapy [4-13].

The use of inverse optimization algorithm in cervical cancer brachytherapy has been reported [14-19]. Although successful in prostate brachytherapy, its use in GYN brachytherapy is still not common, due to various issues which have 
been reported. Chajon et al. [16] reported that a large variation of dwell times was observed with IPSA for tandem ovoid application and suggested the use of help structures to reduce the variation. Further it was reported by Trnkova et al. [19] that Hybrid Inverse Planning Optimization (HIPO) algorithm produces plans without high dose regions for both T/R and Vienna applicator and concluded that modulation restriction, which is used in HIPO plays an important role in obtaining a distribution, without much of heterogeneity as compared to IPSA. A recent study by Mavroids et al. [20] for prostate brachytherapy investigates the use of modulation restriction and concludes that modulation restricted optimization gives similar results as compared to optimization without modulation restriction; however, it may slightly improve the dose distributions.

Hence, it appears that there are a few issues with inverse planning used especially in intracavitary brachytherapy, which need to be clearly understood how to perform it under various situations and how much the result deviates from the clinically acceptable standard, i.e. the standard loading pattern or manual optimization, before implementing into clinical practice. We recently purchased a commercially available inverse planning algorithm (Inverse Planning Simulated Annealing - IPSA) for our clinic. As part of the commissioning process, we carried out a detailed study comparing the dosimetric outcome of plans generated by IPSA with manual optimization. To study the effect of modulation restriction we made IPSA plans with and without help structures for two types of applicators used in our clinic which are the tandem/ovoid $(\mathrm{T} / \mathrm{O})$ and Vienna applicators.

The purpose of this study is to report our experience while we objectively compared the dosimetric outcome of IPSA, with the focus on the use of help structures during optimization for $\mathrm{T} / \mathrm{O}$ and Vienna applicators.

\section{Material and methods}

The data of thirty three patients who underwent MR Image based HDR intracavitary brachytherapy for cervix cancer was selected for this retrospective study. There were eleven IIIB, fourteen IIB, two IIA and one IB2 patients. A dose of 50 Gy was given as external beam radiotherapy dose. Out of 33 patients, 23 patients were treated with intracavitary approach $(\mathrm{I} / \mathrm{C})$ using Nucletron standard $\mathrm{T} / \mathrm{O}$ $\mathrm{CT} / \mathrm{MR}$ compatible applicators with tandem lengths of 5 or $6 \mathrm{~cm}$ and ovoid diameters of 2 or $1.5 \mathrm{~cm}$. Ten patients were treated with combined intracavitary and interstitial approach (I/C + I/S) with Vienna applicator [21]. All patients were transferred to the MRI scanner soon after completion of the application in operation theatre. The inhouse built MRI dummies filled with water were inserted in the catheters to visualize central tandem, ovoid and ring in the scans. T2 weighted fast spin echo MRI scans (GE, Signa, Excite, $1.5 \mathrm{~T}^{\circledR}$ ) were obtained in the axial, coronal, and sagittal orientations. The scans were taken with $3 \mathrm{~mm}$ thickness and $0 \mathrm{~mm}$ spacing. These scans were transferred to the Oncentra contouring workstation (Nucletron, Netherlands ${ }^{\circledR}$ ) where high risk clinical target volume (HR CTV), bladder, rectum and sigmoid were contoured in accordance with GEC ESTRO recommendation [22]. Direct applicator reconstruction was carried out on the MR images using multi-planner reconstruction [23]. The dose computation algorithm was based on TG 43 as recommended by the American Association of Physicists in Medicine (AAPM) [24]. Treatment planning was carried out with the PLATO Sunrise planning system (Nucletron, Netherlands ${ }^{\circledR}$ ).

Three plans were generated for T/O and Vienna applicator: manual optimized plan (MOPT), IPSA without help structures (IPSA_woHS) and IPSA with help structures (IPSA_wHS). Prescription dose of 7 Gy was used for these three plans.

\section{MOPT}

MOPT plans were generated by applying manual optimization to the standard plan which was based on Fletcher standard loading system. Manual optimization, also called graphical optimization, was done by altering dwell times/dwell weights or by dragging the isodose lines using mouse in MRI images. The dwell positions outside the HR CTV were not switched off during manual optimization to retain the pear shaped distribution. The dwell positions contributing to the coverage was increased and the dwell positions contributing to the dose to OARs were reduced appropriately. In case of the Vienna applicator, the loading of the needles were kept at about $15-20 \%$ as compared to the tandem and the ring [21]. The dose volume constraints used for this plan were as follows: HR CTV: $\mathrm{D}_{90}=7.0 \mathrm{~Gy}, \mathrm{D}_{2 \mathrm{cc}}$ of bladder $\leq 5.6 \mathrm{~Gy}$ and $\mathrm{D}_{2 \mathrm{cc}}$ of rectum and sigmoid $\leq 4.4 \mathrm{~Gy}$. After optimization, the DVH and the dose distribution were analyzed. If the DVH parameters did not meet the required criteria, further changes were made to the dwell times and the DVH analysis was performed again until the best solution, meeting all the criteria, was achieved.

\section{Inverse plans}

Two inverse plans were completed: IPSA_woHS and IPSA_wHS. For IPSA_woHS, HS around tandem, ovoid/ring and needles were ignored during inverse optimization, whereas for IPSA_wHS they were taken into account. The dose volume objectives used for inverse plan are shown in Table 1. All the plans were started with the dose volume objectives presented in the table. Based on the results of the previous plan, the objectives were changed by trial and error until an optimal plan was obtained. The dose volume constraints used for IPSA were similar to MOPT.

\section{Plan evaluation}

The DVH was calculated with the upper dose limit factor of 3 and 100,000 sampling points in the volume of interest. The dose volume parameters recommended by GEC ESTRO GYN were analyzed for comparison: $V_{100}$ (percentage of volume covering $100 \%$ of the dose), $\mathrm{D}_{90}$ (dose to $90 \%$ of HR CTV), $\mathrm{D}_{2 \mathrm{cc}}$ and $\mathrm{D}_{0.1 \mathrm{cc}}$ (minimum dose to the most exposed 2 cc \& 0.1 cc of bladder, rectum and sigmoid) [22]. For the evaluation of high dose region $V_{200}$, the volume receiving $200 \%$ of the prescription dose was also evaluated. Dose to point-A and TRAK (Total Reference Air Kerma) 
Table 1. Dose volume objectives used for IPSA plans

\begin{tabular}{|c|c|c|c|c|c|c|c|c|c|c|}
\hline VOI & $\begin{array}{c}\text { Margin } \\
(\mathrm{mm}) \\
\text { dose } \\
\text { control }\end{array}$ & $\begin{array}{l}\text { Margin } \\
(\mathrm{mm}) \\
\text { catheter } \\
\text { activation }\end{array}$ & $\begin{array}{l}\text { Min } \\
\text { surface } \\
\text { dose } \\
\text { weight }\end{array}$ & $\begin{array}{c}\text { Min } \\
\text { surface } \\
\text { dose } \\
\text { (cGy) }\end{array}$ & $\begin{array}{c}\text { Max } \\
\text { surface } \\
\text { dose } \\
\text { (cGy) }\end{array}$ & $\begin{array}{l}\text { Max } \\
\text { surface } \\
\text { dose } \\
\text { weight }\end{array}$ & $\begin{array}{l}\text { Min } \\
\text { volume } \\
\text { dose } \\
\text { weight }\end{array}$ & $\begin{array}{l}\text { Min } \\
\text { volume } \\
\text { dose } \\
\text { (cGy) }\end{array}$ & $\begin{array}{c}\text { Max } \\
\text { volume } \\
\text { dose } \\
\text { (cGy) }\end{array}$ & $\begin{array}{c}\text { Max } \\
\text { volume } \\
\text { dose } \\
\text { weight }\end{array}$ \\
\hline HR CTV & 5 & 2 & 100 & 700 & 750 & 100 & 150 & 700 & 750 & 150 \\
\hline Help structure & 0 & 0 & 20 & 1500 & 2700 & 5 & 20 & 1500 & 2700 & 5 \\
\hline Bladder & 0 & 0 & 0 & 500 & 500 & 30 & 0 & 500 & 500 & 30 \\
\hline Rectum & 0 & 0 & 0 & 420 & 420 & 20 & 0 & 420 & 420 & 20 \\
\hline Sigmoid & 0 & 0 & 0 & 420 & 420 & 30 & 0 & 420 & 420 & 30 \\
\hline
\end{tabular}

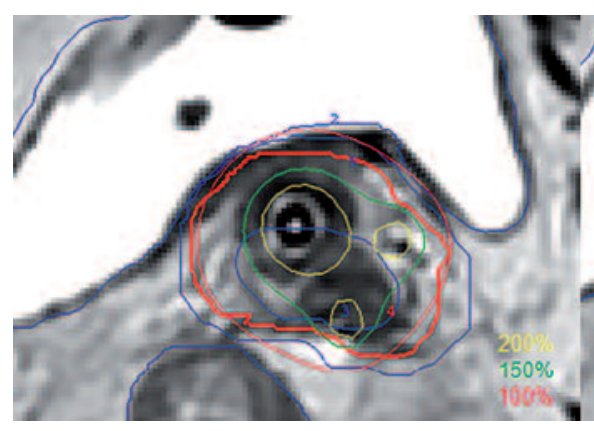

MOPT

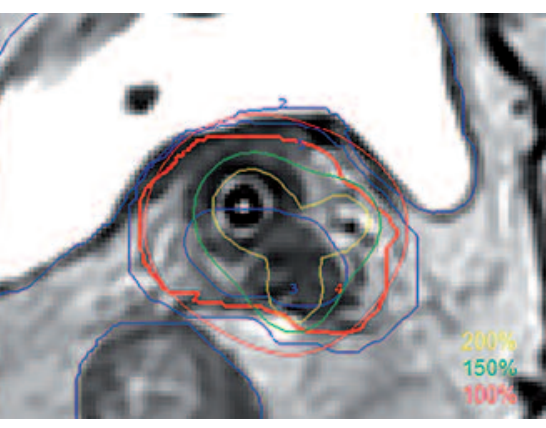

IPSA woHS

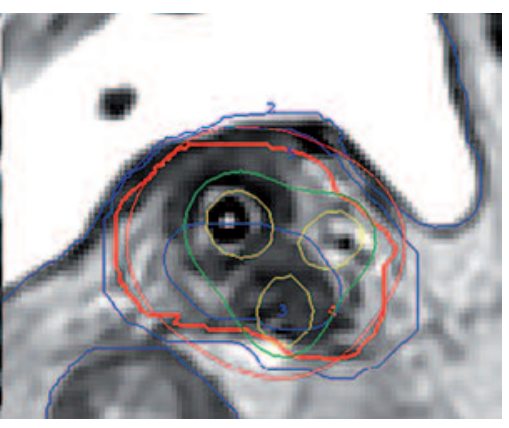

IPSA wHS

Fig. 1. The isodose distribution of a representative patient for various plans, MOPT, IPSA woHS, and IPSA wHS using Vienna applicator

were documented. Conformity index (COIN) was calculated using the following formula:

$\mathrm{COIN}=\left[\left\{V_{p t v, r e f} / V_{p t v}\right\} \times\left\{V_{p t v, r e f} / V_{r e f}\right\}\right]$

where $V_{\text {pto, ref }}$ - target volume received by reference isodose line, $V_{\text {pto }}$ - target volume, $V_{\text {ref }}$ - volume of the implant received by reference isodose line. The data was analyzed using paired sample t-test. The test was performed with a significance level of $5 \%$.

\section{Results}

The isodose distribution of Vienna applicator of a representative patient for various plans is presented in Fig. 1.

\section{T/O applicator}

Table 2 illustrates the dose volume parameters for the HR CTV and OARs. No significant variation was observed with dose volume parameters for HR CTV among the three plans. Similarly, IPSA did not offer significant improvement in sparing the OARs, as compared to MOPT, except for the bladder dose. The $\mathrm{D}_{2 c c}$ bladder dose was significantly less in inverse plans as compared to MOPT plan $(p=0.001)$. The dose to rectum was within tolerance limits $(3.9 \pm 1.2 \mathrm{~Gy})$ in MOPT, but an increased dose was observed in both inverse plans, though not significantly (0.26). It was observed that $\mathrm{V}_{200}$ and $\mathrm{V}_{400}$ was significantly high in both the IPSA plans as compared to MOPT, however in IPSA_wHS, reduced volume was observed and the $p$-value was towards non sig-

Table 2. Dose volume parameters of HR CTV and OARs for T/O applicator

\begin{tabular}{|c|c|c|c|c|c|c|c|}
\hline & $\begin{array}{c}\text { Dose volume } \\
\text { parameter }\end{array}$ & MOPT & IPSA_woHS & IPSA_wHS & $\begin{array}{c}p \\
\text { MOPT vs. WoHS }\end{array}$ & $\begin{array}{c}p \\
\text { MOPT vs. wHS }\end{array}$ & $\begin{array}{c}p \\
\text { wHS vs. woHS }\end{array}$ \\
\hline \multirow[t]{8}{*}{ HR-CTV } & Volume (cc) & \multicolumn{6}{|c|}{$41 \pm 12$} \\
\hline & $\mathrm{D}_{90}(\mathrm{~Gy})$ & $6.2 \pm 0.9$ & $6.1 \pm 0.5$ & $6.0 \pm 0.4$ & 0.19 & 0.22 & 0.25 \\
\hline & V100 (\%) & $85 \pm 7$ & $86.5 \pm 3.4$ & $86.0 \pm 4.1$ & 0.54 & 0.50 & 0.64 \\
\hline & $\mathrm{V}_{200 \mathrm{CC}}$ & $11.2 \pm 4.3$ & $15 \pm 1.9$ & $13.7 \pm 1.9$ & 0.000 & 0.001 & 0.01 \\
\hline & $\mathrm{V}_{400} \mathrm{Cc}$ & $3.1 \pm 1.8$ & $7.6 \pm 1.5$ & $5.4 \pm 1$ & 0.000 & 0.001 & 0.01 \\
\hline & Point A (Gy) & $6.6 \pm 1.9$ & $6.7 \pm 1.4$ & $6.7 \pm 0.9$ & 0.22 & 0.21 & 0.30 \\
\hline & TRAK & $0.48 \pm 09$ & $0.42 \pm 08$ & $0.43 \pm 06$ & 0.001 & 0.000 & 0.30 \\
\hline & COIN & $0.34 \pm 08$ & $0.42 \pm 08$ & $0.41 \pm 05$ & 0.000 & 0.000 & 0.35 \\
\hline Bladder & $D_{2 c c}(G y)$ & $6.5 \pm 1.3$ & $6.0 \pm 1.4$ & $6.1 \pm 1.4$ & 0.001 & 0.001 & 0.29 \\
\hline Rectum & $D_{2 c c}(G y)$ & $3.9 \pm 1.2$ & $4.1 \pm 1.4$ & $4.0 \pm 1.4$ & 0.26 & 0.54 & 0.50 \\
\hline Sigmoid & $D_{2 c c}(G y)$ & $4.9 \pm 1.1$ & $4.8 \pm 1.3$ & $4.8 \pm 1.3$ & 0.40 & 0.45 & 0.59 \\
\hline
\end{tabular}


Table 3. Dose volume parameters of HR CTV and OARs for Vienna applicator

\begin{tabular}{|c|c|c|c|c|c|c|c|}
\hline & $\begin{array}{l}\text { Dose volume } \\
\text { parameter }\end{array}$ & MOPT & IPSA_woHS & IPSA_wHS & $\begin{array}{c}p \\
\text { MOPT vs. woHS }\end{array}$ & $\begin{array}{c}p \\
\text { MOPT vs. wHS }\end{array}$ & $\begin{array}{c}p \\
\text { wHS vs. woHS }\end{array}$ \\
\hline \multirow[t]{8}{*}{ HR-CTV } & Volume (cc) & \multicolumn{6}{|c|}{$39 \pm 14$} \\
\hline & $\mathrm{D}_{90}(\mathrm{~Gy})$ & $7.6 \pm 0.6$ & $7.8 \pm 0.4$ & $7.7 \pm 0.2$ & 0.001 & 0.002 & 0.38 \\
\hline & $V_{100}(\%)$ & $93.8 \pm 3.3$ & $98 \pm 1.8$ & $98.5 \pm 2$ & 0.005 & 0.006 & 0.64 \\
\hline & V200 CC & $13.5 \pm 0.9$ & $21 \pm 0.5$ & $15.8 \pm 0.4$ & 0.000 & 0.001 & 0.003 \\
\hline & $\mathrm{V}_{400} \mathrm{CC}$ & $4.3 \pm 0.5$ & $12 \pm 0.3$ & $7 \pm 0.3$ & 0.000 & 0.001 & 0.000 \\
\hline & Point A (Gy) & $7.3 \pm 1.9$ & $7.6 \pm 0.5$ & $7.5 \pm 0.4$ & 0.002 & 0.006 & 0.30 \\
\hline & TRAK & $0.43 \pm .06$ & $0.39 \pm .03$ & $0.40 \pm .02$ & 0.001 & 0.001 & 0.30 \\
\hline & COIN & $0.32 \pm .08$ & $0.42 \pm .06$ & $0.43 \pm .04$ & 0.000 & 0.000 & 0.5 \\
\hline Bladder & $\mathrm{D}_{2 \mathrm{cc}}(\mathrm{Gy})$ & $5.4 \pm 0.9$ & $5.0 \pm 0.5$ & $5.1 \pm 0.4$ & 0.000 & 0.001 & 0.25 \\
\hline Rectum & $D_{2 c c}(G y)$ & $3.6 \pm 1$ & $3.7 \pm 0.5$ & $3.6 \pm 0.3$ & 0.51 & 0.52 & 0.51 \\
\hline Sigmoid & $\mathrm{D}_{2 \mathrm{cc}}(\mathrm{Gy})$ & $3.4 \pm 0.8$ & $3.5 \pm 0.6$ & $3.3 \pm 0.5$ & 0.50 & 0.52 & 0.55 \\
\hline
\end{tabular}

nificance. Similarly, the TRAK was significantly lower in IPSA as compared to MOPT ( $p=0.001)$, which may suggest that dose distribution from IPSA plans were customized to the individual tumour shape without maintaining the conventional pear shaped dose distribution as in case of MOPT. In terms of conformity, IPSA plans scores over MOPT plan, as IPSA plans were significantly conformal as compared to MOPT $(p=0.000)$.

\section{Vienna applicator}

The dose volume parameters of HR CTV showed statistical significance in favour of IPSA as compared to MOPT (Table 3). However, it is to be noted that in addition to a considerable improvement in $\mathrm{D}_{90}$ and $\mathrm{V}_{100}$ of HR CTV, the high dose regions $V_{200}$ and $V_{400}$ had also increased significantly in inverse plans as compared to MOPT plans. It is also worth noting that IPSA plans were more reproducible as compared to the MOPT plan, as the standard deviation of all the dose volume parameters of IPSA was smaller as compared to MOPT plans. TRAK and COIN showed statistical significance in favour of IPSA, which could be attributed to the individual adaptation of IPSA to tumour volume, as against standard loading pattern in case of MOPT $(p=0.001)$. However, amongst OARs, only the bladder dose was significantly improved with IPSA, as compared to the rectum and sigmoid. The dose volume parameters of both HR CTV and OARs between IPSA_woHS and IPSA_wHS were found insignificant for all the dose volume parameters and significant for the high dose regions, $V_{200}$ and $V_{400}$.

\section{Loading pattern and treatment time}

For the T/O applicator, the ratio of dwell time of tandem to ovoid $\left(\mathrm{T}_{\mathrm{T} / \mathrm{O}}\right)$ was found to be $0.93 \pm 0.2,0.64 \pm 0.15,0.66$ \pm 0.17 for MOPT, IPSA_woHS and IPSA_wHS, respectively. For both the inverse plans, the ratio showed significant deviation as compared to MOPT plan. On the other hand, for the Vienna applicator, the ratio of dwell time of tandem to $\operatorname{ring}\left(\mathrm{T}_{\mathrm{T} / \mathrm{R}}\right)$ was found to be $1.02 \pm 0.15,0.94 \pm 0.1,0.98 \pm$ 0.08 for MOPT, IPSA_woHS and IPSA_wHS, respectively. However, the ratio of total time of needles to the tandem
$\left(\mathrm{T}_{\mathrm{N} / \mathrm{T}} \%\right)$ was found to be $14 \pm 2.5,53 \pm 9,22 \pm 6$ for MOPT, IPSA_woHS and IPSA_wHS, respectively (Table 4), which implies that in IPSA_woHS the dwell time in needles were half of the dwell time in the tandem, while in MOPT the needles were loaded in $14 \%$ and in IPSA_wHS it was $22 \%$ of the dwell time of tandem. It was observed that IPSA_woHS had highest contribution from needles, while help structures in IPSA_wHS has significantly reduced the dwell time contribution from the needles $(p=0.000)$. It could be noted that the ratio $T_{T / R}$ is comparable with each other for the three plans for Vienna applicator, while in case of $\mathrm{T} / \mathrm{O}$ applicator both IPSA plans showed significant variation as compared to MOPT, which may be attributed to the involvement of needles. It was observed that the contribution from the needles is highest in IPSA_woHS. Inclusion of the HS in the optimization reduced the contribution of dwell time of the needles in the IPSA_HS plan and was of the same order as the MOPT plan. During manual optimization it was considered that most of the dose came from the intracavitary Vienna applicator with the needles having very low loading only to cover the missing parts of the HR CTV, which was not possible to cover with the intracavitary alone [21].

With respect to individual variation in dwell time for the $\mathrm{T} / \mathrm{O}$ applicator, IPSA resulted in steep variation in the dwell time in the catheters, which resulted in-homogeneous plans, as compared to MOPT. It was also observed that in IPSA, the dwell time next to a critical structure was turned off completely, while long dwell times were noticed where no critical structures were present. Figure $2 a \& 2 b$ shows the individual dwell time of catheters for MOPT, IPSA_woHS and IPSA_wHS plans of a representative patient for $\mathrm{T} / \mathrm{O}$ and Vienna applicator.

\section{Planning time}

The planning time for MOPT plan was 15-20 minute, while planning time for IPSA was about $10 \mathrm{~min}$. Changing the constraints and re-evaluating the dose volume parameters to arrive at an optimal plan was indeed a time consuming factor for IPSA plans as well. However, it took about 20 minutes to arrive at an optimal MOPT plan for a Vien- 
Table 4. The absolute dwell time in seconds of T/O and Vienna applicator for MOPT: Manual plan, wHS: IPSA plan with help structures, woHS: IPSA plan without help structures

\begin{tabular}{|c|c|c|c|c|c|c|}
\hline \multicolumn{4}{|c|}{ T/O applicator } & \multicolumn{3}{|c|}{$p$ value } \\
\hline Dwell time & MOPT & woHS & wHS & MOPT vs. woHS & MOPT vs. wHS & wHS vs. woHS \\
\hline Tandem (sec) & $340 \pm 38$ & $253 \pm 65$ & $270 \pm 45$ & 0.000 & 0.040 & 0.015 \\
\hline Rt ovoid (sec) & $180 \pm 26$ & $228 \pm 71$ & $210 \pm 38$ & 0.003 & 0.025 & 0.021 \\
\hline Lt ovoid (sec) & $185 \pm 45$ & $170 \pm 52$ & $198 \pm 54$ & 0.005 & 0.010 & 0.000 \\
\hline Total time (sec) & $705 \pm 49$ & $651 \pm 81$ & $678 \pm 51$ & 0.003 & 0.020 & 0.008 \\
\hline Total ovoid (sec) & $365 \pm 41$ & $398 \pm 47$ & $408 \pm 49$ & 0.004 & 0.002 & 0.330 \\
\hline \multirow[t]{2}{*}{ Tandem/Total ovoid } & $0.93 \pm 0.2$ & $0.64 \pm 0.15$ & $0.66 \pm 0.17$ & 0.000 & 0.002 & 0.370 \\
\hline & \multicolumn{2}{|c|}{ Vienna applicator } & & & & \\
\hline Tandem (sec) & $361 \pm 29$ & $270 \pm 46$ & $320 \pm 42$ & 0.001 & 0.150 & 0.030 \\
\hline Ring (sec) & $353 \pm 38$ & $286 \pm 69$ & $325 \pm 37$ & 0.002 & 0.180 & 0.028 \\
\hline Needles (sec) & $51 \pm 15$ & $143 \pm 105$ & $70 \pm 39$ & 0.000 & 0.130 & 0.000 \\
\hline Total time (sec) & $765 \pm 59$ & $699 \pm 64$ & $715 \pm 59$ & 0.003 & 0.100 & 0.007 \\
\hline Needles/Tandem time (\%) & $14 \pm 2.5$ & $53 \pm 9$ & $22 \pm 6$ & 0.000 & 0.150 & 0.000 \\
\hline Tandem/Ring & $1.02 \pm 0.15$ & $0.94 \pm 0.1$ & $0.98 \pm 0.08$ & 0.170 & 0.200 & 0.350 \\
\hline
\end{tabular}

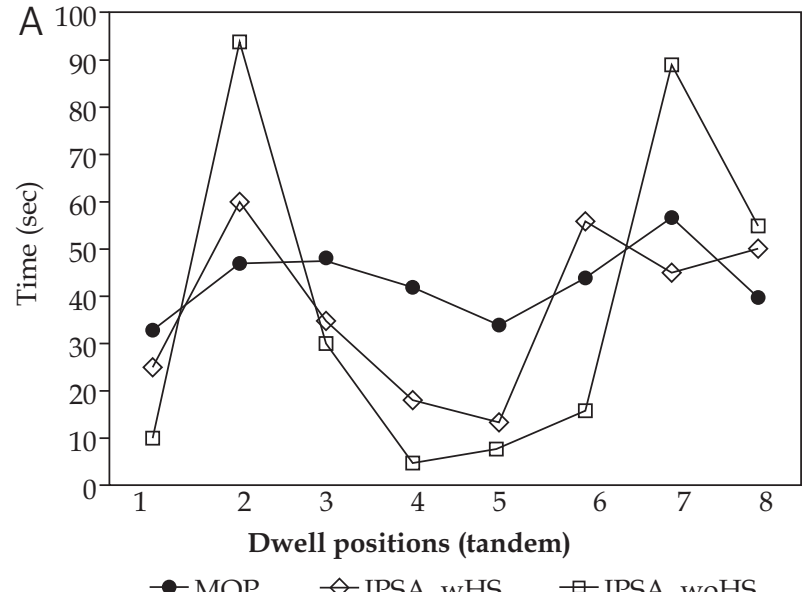

Fig. 2A. Individual dwell time of a tandem for three plans which are: manual optimized plan (MOPT), IPSA plan done with help structures (IPSA_wHS), and IPSA plan without help structures (IPSA_wsHS) for T/O applicator

na applicator by an experienced physicist. Changing the individual dwell weights, manually dragging the isodose lines using a mouse and evaluation of dose volume parameters was a time consuming process during MOPT plans. Drawing of help structures was also found to be a time consuming process, although we did not quantify the time.

\section{Discussion}

In intracavitary brachytherapy, inverse planning is not a popular concept due to various factors. One is the reluctance to change from traditional practice, which produced excellent results as the pear shaped distribution has been used for decades and resulted in good clinical outcomes $[1,2]$. Inverse planning in intracavitary brachytherapy is always seen with a doubt, due to different factors. The clin-

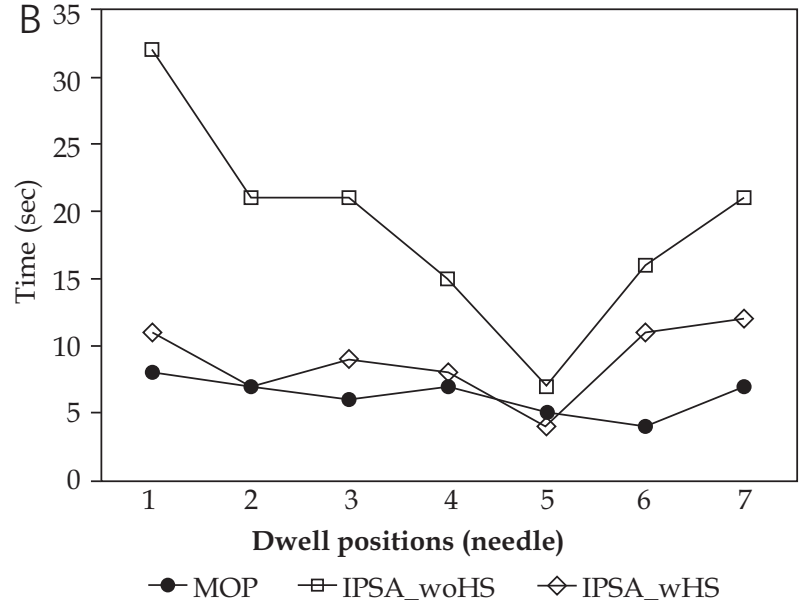

Fig. 2B. Individual dwell time of a needle for three plans viz manual optimized plan (MOPT), IPSA plan done with help structures (IPSA_wHS), and IPSA plan without help structures (IPSA_wsHS) for Vienna applicator

ical implication of changing the standard loading pattern is not known. Similarly, the variation of dwell time across the catheters due to the absence of modulation restriction in certain algorithm was debated [16, 19, 20, 25]. Although, it has been concluded by a few investigators that IPSA produces superior dose distribution in terms of improved and highly conformal target coverage and reduced dose to OARs, it is still unclear whether the variation of dwell time across the catheters due to the absence of modulation restriction plays a role in toxicities. While this information is missing, it is still believed that pear shaped distribution should be maintained as far as possible. Chajon et al. have proposed defining help structures around the applicator to obtain homogenous dwell time distribution, and to overcome the problem of variation of dwell time while using IPSA, with GYN application [16]. However, the 
generation of such dummy structures would be time consuming in a busy clinical centre especially in case of IC + IS approach. Hence, we carried out a study to find out whether the help structures really did help in reducing the variation of dwell time as well as its impact on the dose volume parameters.

The results of the present study indicate that inverse plans in general, produced significant sparing of bladder while maintaining the HR CTV coverage. IPSA has not made any significant variation in the dose to rectum and sigmoid for both $\mathrm{T} / \mathrm{O}$ and Vienna applicator. However, the high dose regions V200 and V400 were significantly higher in IPSA as compared to MOPT. It was found that high dose regions were significantly higher in IPSA_woHS than MOPT, which agrees with other investigators findings $[16,26]$. Detailed analysis of each patient's planned isodose distribution showed that, in case of $\mathrm{T} / \mathrm{O}$ applicator, the majority of high dose regions were found around the tandem and the ovoids. In most of the patients, high dose regions were also found next to a dwell position in which dwell weight was nearly zero to reduce its contribution to an OAR. It was observed that IPSA switches off certain dwell positions which are located close to posterior bladder and anterior rectal wall that is close to the applicator. However, the dwell weight next to such a dwell position would be very high to compensate for the missing region of HR CTV, that increases the localized hotspots. Inclusion of HS and a heavy penalty for HS during optimization had reduced the volume of the high dose region around the tandem and the ovoid significantly with reduction to the variation of the dwell time which would be discussed later. On the other hand, in case of Vienna applicator, the hot spots were found equally around the needles as that of tandem and ring. It was also found that the dwell weights of the tandem/ring and the needles were almost equal. In fact in few patients, where the tandem was close to the bladder and sigmoid, the dwell weights of the needles were higher as compared to tandem and ring. Here the dwell time near a sigmoid and the bladder was turned off, and the dwell weight of the needles was higher. In such cases, region of V200 and V400 was present around the needles as a continuous volume. When HS were included in the optimization with higher penalties for the HS of the needles, the high dose regions around the needles were reduced. The continuous volume of high dose region around the needle would break to form islands around the dwell positions. Further optimization with much heavier penalty to HS of the needles reduced the size of the hot spots around the needles. Although the volume of V200 and V400 was not higher in the case of Vienna applicator as compared to the T/O applicator, it was found that the spatial location of high dose regions was shifted towards the needles or distributed equally among the tandem, ring and the needles.

The ratio of $\mathrm{T}_{\mathrm{T} / \mathrm{O}}$ and $\mathrm{T}_{\mathrm{T} / \mathrm{R}}$ did not show any significant variation among the IPSA plans for both $\mathrm{T} / \mathrm{O}$ and Vienna applicator. However, $\mathrm{T}_{\mathrm{N} / \mathrm{T}}$ was as high as $53 \%$ for IPSA woHS, while it was $22 \%$ for IPSA_wHS. This may imply that the benefit of using the help structures during optimization is superior when the number of catheters is more than two/three catheters as compared to the T/O and T/R applicators. Hence, it may be considered that while using com- bined intracavitary and interstitial (IC + IS) approach, help structures may be used during optimization and need not be used with intracavitary application as the benefit is negligible both in terms of dosimetric parameters and dwell time. It is not very clear as to why, during the IC approach, the help structures are not as beneficial as with the IC + IS approach. However, it may be attributed to the number of degrees of freedom associated with the interstitial approach. Caution is therefore required while using IPSA in case of IC + IS approach, as it may lead to overloading of needles and variation of dwell time, especially in the needles. We found that this approach of drawing help structures in case of Vienna applicator is not very practical for routine clinical work, as it is a time consuming process.

In a recent publication, Trnkova et al. has compared HIPO and IPSA algorithm and concluded that HIPO optimization offers better dose distribution than IPSA, in terms of controlling the high dose regions, as HIPO employs dwell time gradient restriction [26]. It is still unclear, how this is going to translate clinically. However, recent clinical results justify the use of IPSA in the clinics [27]. Kim et al. had presented clinical outcomes of image-guided brachytherapy using IPSA high dose rate brachytherapy boost of 51 patients of cervical cancer with two year follow up. They concluded that IPSA HDR brachytherapy is well tolerated and achieves excellent local control of disease. Though, it should be noted that the study was based on IC and not IC + IS approach. The results of the current study also justify the use of IPSA in the clinics for T/O application. Nevertheless, extreme caution is required in controlling the high dose regions. The findings of the present study show that drawing of HS around the tandem, and ovoids help to reduce the hotspot around the catheters. The benefit of using the HS is more in Vienna applicator as compared to T/O applicator. However, in our experience, producing IPSA plans for Vienna applicator that generate a distribution, which does not deviate very much from the traditional shape is too labour intense and depends on the experience of the planner. Although it produces a similar dose volume parameters as compared to MOPT after optimization using HS, the high dose regions around the needles and the variation of the dwell time across the catheters makes it a nonviable solution in the daily clinical routine. On the other hand, in our experience, MOPT plans are much easier to produce, as we generally start with $85 \%$ loading to T/R and $15 \%$ to needles. The loading of the needles would be increased slowly based on the dose volume parameters of the HR CTV and the OARs. This way the planner is aware that the loading is comparable to traditional loading pattern and the contribution from the needles are just adequate enough to cover the missing lateral part of the HR CTV. In case of Vienna applicator, the clinical consequences of the presence of high dose regions especially around the needles are unknown since they were nonexistent in time tested traditional loading pattern and manual after loading.

In a recent publication comparing manual and inverse plans for intracavitary and interstitial applicator, it was concluded that IPSA overloaded the needles. The findings of the present study are in agreement with the results of Trnková et al. [26]. They further reported that HIPO was able to achieve a similar dose distribution compared to ma- 
nual planning with the restriction of high dose regions and reduced loading time of needles. This was possible due to the dwell time gradient restriction offered by HIPO. In the current study, HS were introduced to simulate the dwell time gradient restriction, and resulted in improved parameters with respect to high dose regions. Mavroidis et al. has carried out a radiobiological evaluation of the influence of dwell time modulation restriction in prostate brachytherapy using HIPO, and concluded that modulation restricted optimization gives on average similar results with the optimization without modulation restriction [20]. Optimization with modulation restriction gives only a minor improvement in the effectiveness of plans. While this is true for prostate brachytherapy, further investigation may be required for GYN brachytherapy, where traditionally, the high dose is restricted inside the uterus and may emphasize the need for further investigation of the clinical implication of modulation restriction.

\section{Conclusions}

IPSA plans were compared with manual plan for $\mathrm{T} / \mathrm{O}$ and Vienna applicator and it was found that IPSA produced similar plans with respect to HR CTV coverage and sparing of OARs, but with higher volumes of high dose regions. IPSA also produced conformal plans as compared to MOPT. For T/O applicator, it was found that the use of HS did not make any impact in the dose volume parameters and in the loading time of tandem and ovoids, however in case of Vienna applicator, inclusion of HS in the optimization made a significant impact in the loading of needles and reduced the high dose regions around the needles. Without the HS, the treatment time in the needles was high, which was significantly reduced when HS were included.

\section{References}

1. Patel FD, Rai B, Mallick I et al. High-dose-rate brachytherapy in uterine cervical carcinoma. Int J Radiat Oncol Biol Phys 2005; 62: $125-130$.

2. Hareyama M, Sakata K, Oouchi A et al. High-dose-rate versus low-dose-rate intracavitary therapy for carcinoma of the uterine cervix: a randomized trial. Cancer 2002; 94: 117-124.

3. Potter R et al. Clinical impact of MRI assisted dose volume adaptation and dose escalation in brachytherapy of locally advanced cervix cancer. Radiother Oncol 2007; 83: 148-155.

4. Lessard E, Pouliot J. Inverse planning anatomy-based dose optimization for HDR brachytherapy of the prostate using fast simulated annealing algorithm and dedicated objective function. Med Phys 2001; 28: 773-779.

5. Lachance B, Beliveau-Nadeau D, Lessard E et al. Early clinical experience with anatomy-based inverse planning dose optimization for high-dose-rate boost of the prostate. Int J Radiat Oncol Biol Phys 2002; 54: 86-100.

6. Kolkman-Deurloo IK, Deleye XG, Jansen PP et al. Anatomy based inverse planning in HDR prostate brachytherapy. Radiother Oncol 2004; 73: 73-77.

7. Pouliot J, Kim Y, Lessard E, et al. Inverse planning for HDR prostate brachytherapy used to boost dominant intraprostatic lesions defined by magnetic resonance spectroscopy imaging. Int J Radiat Oncol Biol Phys 2004; 59: 1196-1207.

8. Citrin D, Ning H, Guion P et al. Inverse treatment planning based on MRI for HDR prostate brachytherapy. Int J Radiat Oncol Biol Phys 2005; 61: 1267-1275.
9. Lessard E, Kwa SL, Pickett B et al. Class solution for inversely planned permanent prostate implants to mimic an experienced dosimetrist. Med Phys 2006; 33: 2773-2782.

10. Raben A, Sammons S, Sim S et al. Initial comparison of inverse optimization, modified peripheral technique, and geometric optimization as real-time intraoperative computer planning options for permanent seed implantation of the prostate. Brachytherapy 2007; 6: 238-245.

11. Jacob D, Raben A, Sarkar A et al. Anatomy-Based Inverse Planning Simulated Annealing Optimization in High-Dose-Rate Prostate Brachytherapy: Significant Dosimetric Advantage Over Other Optimization Techniques. Int J Radiat Oncol Biol Phys 2008; 72: 820-827.

12. Kim Y, Hsu IC, Lessard E et al. Class solution in inverse planned HDR prostate brachytherapy for dose escalation of DIL defined by combined MRI/MRSI. Radiother Oncol 2008; 88: 148-155.

13. Morton GC, Sankreacha R, Halina P et al. A comparison of anatomy-based inverse planning with simulated annealing and graphical optimization for high dose- rate prostate brachytherapy. Brachytherapy 2008; 7: 12-16.

14. Lessard E, Hsu IC, Pouliot J. Inverse planning for interstitial gynecologic template brachytherapy: truly anatomy-based planning. Int J Radiat Oncol Biol Phys 2002; 54: 1243-1251.

15. Dewitt KD, Hsu IC, Speight J et al. 3D inverse treatment planning for the tandem and ovoid applicator in cervical cancer. Int J Radiat Oncol Biol Phys 2005; 63: 1270-1274.

16. Chajon E, Dumas I, Touleimat $M$ et al. Inverse planning approach for 3-D MRI-based pulse-dose rate intracavitary brachytherapy in cervix cancer. Int J Radiat Oncol Biol Phys 2007; 69: 955-961.

17. Kubicky CD, Yeh BM, Lessard E et al. Inverse planning simulated annealing for magnetic resonance imaging-based intracavitary high-dose-rate brachytherapy for cervical cancer. Brachytherapy 2008; 7: 242-247.

18. Kim Y, Hsu IC, Lessard E et al. Class solution in inverse planned HDR prostate brachytherapy for dose escalation of DIL defined by combined MRI/MRSI. Radiother Oncol 2008; 88: 148-155.

19. Trnkova P, Potter R, Baltas D et al. New inverse planning technology for image-guided cervical cancer brachytherapy: description and evaluation within a clinical frame. Radiother Oncol 2009; 93: 331-340.

20. Mavoroids P, Katsileri Z, Kefala V et al. Radiobiological evaluation of the influence of dwell time restriction in HIPO optimized HDR prostate brachytherapy implants. J Contemp Brachyther 2010; 2: 117-128.

21. Kirisits C, Lang S, Dimopoulos J et al. The Vienna applicator for combined intracavitary and interstitial brachytherapy of cervical cancer: design, application, treatment planning, and dosimetric results. Int J Radiat Oncol Biol Phys 2006; 65: 624-630.

22. Haie-Meder C et al. Recommendations from Gynaecological (GYN) GEC-ESTRO Working Group (I): concepts and terms in 3D image based 3D treatment planning in cervix cancer brachytherapy with emphasis on MRI assessment of GTV and CTV. Radiother Oncol 2005; 74: 235-245.

23. Berger D, Demopoulos J, Potter R et al. Direct reconstruction of the Vienna applicator on MR images. Radiother Oncol 2009; 93: 347-351.

24. Rivard MJ, Coursey BM, DeWerd LA et al. Update of AAPM Taskgroup No. 43 report. A revised AAPM protocol for Brachytherapy dose calculations. Med Phys 2004; 31: 663-674.

25. Jamema SV, Kirisits C, Mahantshetty U et al. Comparison of DVH parameters and loading patterns of standard loading, manual and inverse optimization for intracavitary brachytherapy on a subset of tandem/ovoid cases. Radiother Oncol 2009; 97: 501-506. 
26. Trnková P, Baltas D, Karabis A et al. A detailed dosimetric comparison between manual and inverse plans in HDR intracavitary /interstitial cervical cancer Brachytherapy. J Contemp Brachyther 2010; 2: 163-170.

27. Kim DH, Wang-Chesebro A, Weinberg V et al. High-dose rate brachytherapy using inverse planning simulated annealing for locoregionally advanced cervical cancer: a clinical report with 2-year follow-up. Int J Radiat Oncol Biol Phys 2009; 75: 1329-1334. 